\title{
Detecting gravitational waves from accreting neutron stars
}

\author{
Anna L. Watts ${ }^{\mathrm{a}, *}$, Badri Krishnan ${ }^{\mathrm{b}}$ \\ a Astronomical Institute "Anton Pannekoek", University of Amsterdam, Kruislaan 403, 1098 SJ Amsterdam, The Netherlands \\ b Albert-Einstein-Institut, Max-Planck-Institut für Gravitationsphysik, Am Mühlenberg 1, 14476 Golm, Germany
}

Received 9 October 2008; received in revised form 11 December 2008; accepted 12 January 2009

\begin{abstract}
The gravitational waves emitted by neutron stars carry unique information about their structure and composition. Direct detection of these gravitational waves, however, is a formidable technical challenge. In a recent study we quantified the hurdles facing searches for gravitational waves from the known accreting neutron stars, given the level of uncertainty that exists regarding spin and orbital parameters. In this paper we reflect on our conclusions, and issue an open challenge to the theoretical community to consider how searches should be designed to yield the most astrophysically interesting upper limits. With this in mind we examine some more optimistic emission scenarios involving spin-down, and show that there are technically feasible searches, particularly for the accreting millisecond pulsars, that might place meaningful constraints on torque mechanisms. We finish with a brief discussion of prospects for indirect detection. (C) 2009 COSPAR. Published by Elsevier Ltd. All rights reserved.
\end{abstract}

Keywords: Accretion; Accretion disks; Gravitational waves; Stars: neutron; Stars: rotation; X-rays: binaries; X-rays: bursts

\section{Introduction}

The last few years have seen the commissioning of an advanced and highly sensitive network of interferometric gravitational wave detectors: LIGO,${ }^{1}$ GEO- $600,{ }^{2}$ VIRGO ${ }^{3}$ and TAMA. ${ }^{4}$ These observatories are now at or very close to their initial design sensitivity, and order of magnitude improvements in performance are expected over the next few years as they are upgraded to incorporate major technological advances.

There is no question that we are on the verge of the first direct detection of these elusive ripples in space-time. When this happens, it will be a stunning confirmation of one of the main predictions of the Theory of General Relativity. It will also open up uncharted territory for astro-

\footnotetext{
${ }^{*}$ Corresponding author.

E-mail address: A.L.Watts@uva.nl (A.L. Watts).

URL: http://www.astro.uva.nl/ awatts/ (A.L. Watts).

${ }^{1} \mathrm{http}: / /$ www.ligo.caltech.edu

${ }^{2} \mathrm{http}: / /$ www.geo600.aei.mpg.de

${ }^{3} \mathrm{http}: / /$ wwwcascina.virgo.infn.it

${ }^{4}$ http://tamago.mtk.nao.ac.jp
}

physicists. A gravitational wave can pass almost unattenuated through matter that would absorb and reemit a photon many millions of times. Gravitational waves will let us study regions of the Universe that have until now remained hidden, illuminating completely new physical processes.

The challenges involved, however, are immense. The amplitudes from even the strongest astrophysical sources are so weak that extricating signals from the noise requires advanced search techniques and, where feasible, signal templates. When source parameters are poorly constrained, searches rapidly become both computationally and statistically untenable. It is here that information from electromagnetic astronomy can improve the efficiency of searches, and make the difference between detecting or not detecting a source.

A major target for current searches is the detection of the continuous periodic signals expected from spinning neutron stars. Emission requires some ellipticity on the star, and theorists have been highly inventive in conceiving of mechanisms that might generate a quadrupole. Possibilities include crustal mountains (Bildsten, 1998; Ushomirsky et al., 2000; Melatos and Payne, 2005; Haskell et al., 2006; 
Payne and Melatos, 2006a; Vigelius et al., 2008), internal magnetic deformation (Katz, 1989; Cutler, 2002; Haskell et al., 2008), and internal r-mode oscillations (Andersson et al., 1999, 2000, 2002; Levin, 1999; Heyl, 2002; Wagoner, 2002; Nayyar and Owen, 2006; Bondarescu et al., 2007). Angular momentum loss via gravitational wave emission is also an attractive way of keeping neutron star spin below the break-up limit of $\sim 1 \mathrm{kHz}$ (Lattimer and Prakash, 2007). Accretion should spin stars up to this limit (Cook et al., 1994), but both the accreting neutron stars and the millisecond radio pulsars (their supposed progeny) spin far more slowly (Hessels et al., 2006). The reason for this discrepancy might very well be gravitational wave emission, as discussed below.

\section{The spin balance scenario}

Motivated by the idea that gravitational wave torque could balance accretion torques, Bildsten (1998) pointed out that accreting neutron stars might be steady beacons of gravitational waves. In the simplest scenario, where a neutron star (mass $M$, radius $R$, distance $d$ ) has a quadrupole moment $Q$ that is stationary in the rotating frame of the star, gravitational waves are emitted at a frequency $v=2 v_{s}$, where $v_{s}$ is the spin frequency. We assume an accretion torque

$N_{a} \approx \dot{M}(G M R)^{1 / 2}$

(where accretion rate $\dot{M}=4 \pi R d^{2} F / G M$ can be estimated from the bolometric flux $F$ ), and a gravitational wave torque

$N_{\mathrm{gw}}=-\frac{4 \pi c^{3} d^{2} v_{s} h_{0}^{2}}{5 G}$

where the gravitational wave amplitude $h_{0}$ associated with the quadrupole $Q$ is defined as in Jaranowski et al. (1998). The assumption of spin balance then gives

$h_{0}=3 \times 10^{-27}\left(F_{-8}\right)^{1 / 2} \frac{R_{10}}{M_{1.4}}\left(\frac{1 \mathrm{kHz}}{v_{s}}\right)^{1 / 2}$

where $\quad F_{-8}=F / 10^{-8} \mathrm{ergs} / \mathrm{cm}^{2} / \mathrm{s}, \quad R_{10}=R / 10 \mathrm{~km} \quad$ and $M_{1.4}=M / 1.4 M_{\odot}$. The stronger the flux, the stronger the predicted gravitational wave signal.

Whether this signal is detectable depends on a number of factors. First, the predicted signal must of course exceed the detectability threshold. This depends on the sensitivity of the detector, and the detection level set by the detector teams (in simplistic terms, one requires the amplitude to exceed that generated by noise processes with a specified degree of probability). However, there are other factors at play. In all of the emission scenarios, gravitational wave emission is related to the spin-frequency of the neutron star. This means that in order to fold long stretches of data (necessary for such weak signals), we require an ephemeris for the spin and orbital parameters. If these parameters are known precisely, only a single template must be searched.
If there is any uncertainty, multiple templates must be searched, impacting both statistics (more templates means more trials, so more chance of throwing up a spurious result) and computational load (which sets feasible integration times).

In a recent study we assessed this scenario for all known accreting neutron stars with some indication of spin rate, generating a realistic assessment of detectability using accurate noise curves and detection statistics for current and planned detectors (Watts et al., 2008). We included in our study the accreting millisecond pulsars (spins and orbital parameters mostly well constrained), the burst oscillation sources (spins known to within a few $\mathrm{Hz}$, orbits not so well constrained), and the twin $\mathrm{kHz}$ QPO sources. Whether the spin of the latter class of sources is actually constrained at all by the separation of the twin $\mathrm{kHz}$ QPO frequencies is still a matter of debate. However, theoretical modeling indicates that there may be some link, and so we included them in our study.

Fig. 1 shows the best case detectability for sources with a given flux, assuming that all spin and orbital parameters are sufficiently well constrained that only one template need be used. Fig. 2 shows typical long-term time-averaged fluxes for the various sources (values from Table 1 of Watts

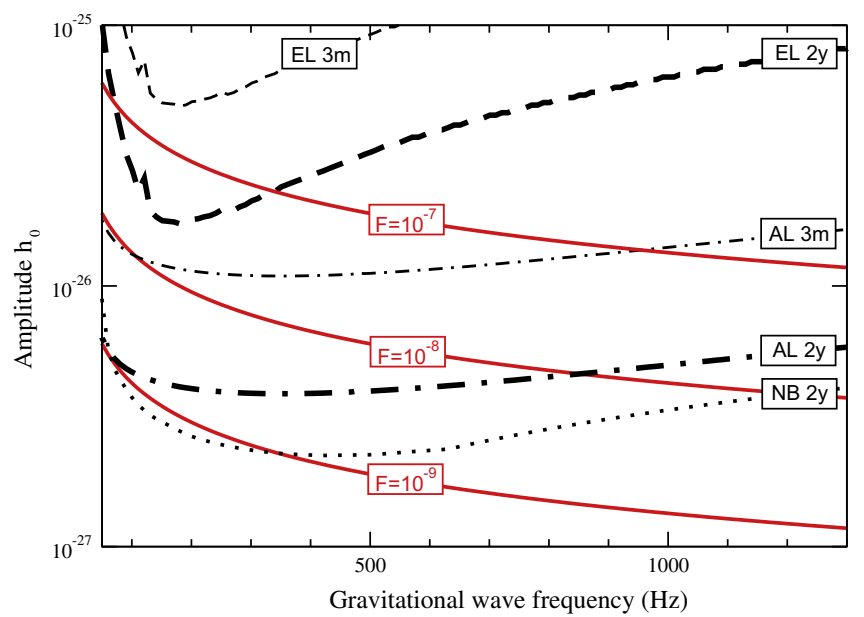

Fig. 1. Detectability for the spin balance scenario, with gravitational wave emission at twice the spin frequency of the neutron star, assuming that only one template need be searched (we have also averaged over all skypositions and pulsar orientations, and chosen thresholds corresponding to a false alarm rate of $1 \%$ and a false dismissal rate of $10 \%$ ). The solid (red) lines show the predicted signal strength for a given X-ray flux $F$ in units of ergs $/ \mathrm{cm}^{2} / \mathrm{s}$. The broken (black) lines show detectability curves for various detector configurations (EL - Enhanced LIGO, AL - Advanced LIGO broad band, NB - Advanced LIGO narrow band envelope) and integration times ( $3 \mathrm{~m}-3$ months, $2 \mathrm{y}-2$ years). Enhanced LIGO is the configuration that will operate during the next LIGO science run in 2009 2010. Advanced LIGO (http://www.ligo.caltech.edu/advLIGO) is the configuration that will operate a few years later after a major upgrade. In each case we have assumed a coherent combination of data from two instruments. For the advanced detectors, we might very well have four instruments (three LIGO and one Virgo) operating in coincidence. This would improve sensitivity by an additional factor of $\sqrt{2}$. (For interpretation of the references to color in this figure legend, the reader is referred to the web version of this paper.) 


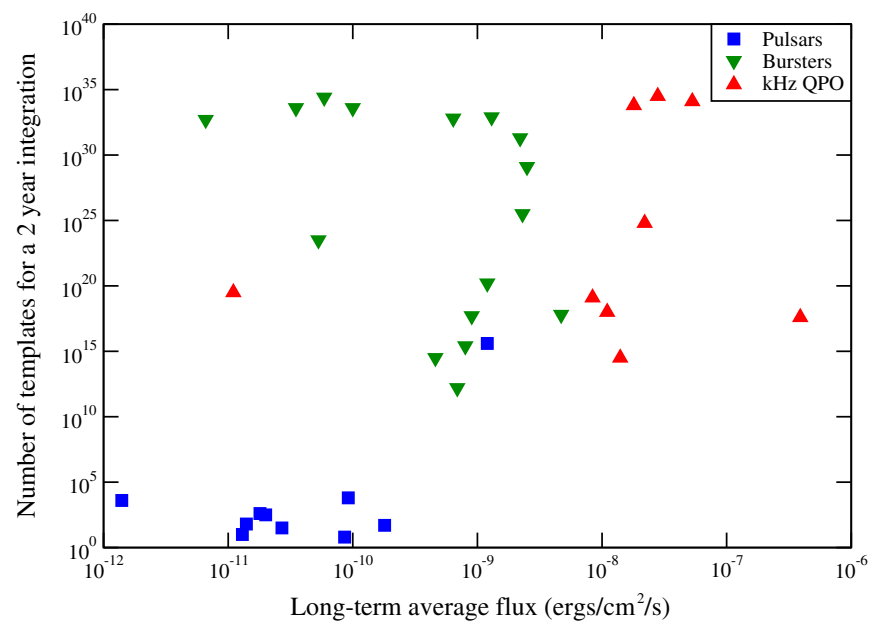

Fig. 2. Factors affecting detectability for the known accreting neutron stars where there is some indication of spin rate: the accreting millisecond pulsars, the burst oscillation sources, and the twin $\mathrm{kHz}$ QPO sources. The plot shows the long-term time-averaged fluxes (compare to the detectability curves in Fig. 1), and the number of templates that would have to be searched for a 2-year integration, given the current uncertainty in spin and orbital parameters for each source (Fig. 1 assumes that only a single template is searched) The best constrained sources tend to be those with the weakest predicted signals (in the spin balance scenario). For reference, we note that $\mathcal{O}\left(10^{15}\right)$ templates is very roughly the largest number of templates that could be searched today using about a year's worth of LIGO data, and large (but still feasible) amounts of computational resources.

et al., 2008). Comparing to Fig. 1 we can see that the accreting millisecond pulsars are simply too weak to be detectable, as are most of the burst oscillation sources (given their frequencies, which tend to be high). ${ }^{5}$ The only sources that are bright enough to be detectable are the $\mathrm{kHz}$ QPO sources. Unfortunately, as is also clear from Fig. 2, these are also the sources with the greatest number of templates to be searched (for full details of the template estimates see Watts et al., 2008). It turns out that the effect on statistics alone, due to the increased number of trials, is enough to push most of the sources below the detectability threshold for Advanced LIGO. Computational considerations impact things still further by reducing feasible integration times. If we search the full frequency band implied by the separation of the twin $\mathrm{kHz}$ QPO frequencies, then only one source remains above the detectability threshold for Advanced LIGO.

The major factor hampering searches for gravitational waves is clearly poor knowledge of spin and orbital parameters for the brighter sources. Efforts to detect accretion-

\footnotetext{
${ }^{5}$ The response times of the gravitational wave torque mechanisms are not well understood. For systems that are transient accretors, like the accreting millisecond pulsars and many of the burst oscillation sources, it may be better to consider the flux in outburst rather than the long-term time-average flux. This scenario, discussed in detail in Watts et al. (2008) is somewhat more optimistic for some sources, since the increase in flux compensates for the short (typically $\sim$ months) outburst times. It is not enough, however, to render any additional sources detectable by Advanced LIGO.
}

powered pulsations from these sources have so far been unsuccessful, and theoretical modeling (Kulkarni and Romanova, 2008) suggests that channeled accretion may simply not occur at the highest accretion rates. Several of the sources exhibit Type I X-ray bursts, and it is possible that burst oscillations (nuclear-powered pulsations seen only during bursts) may yet be detected. It is also possible that theoretical studies may eventually reveal a firm link between $\mathrm{kHz}$ QPO frequency and spin. However, it is perhaps time to consider more radical ways of obtaining spin constraints.

One possibility, for example, would be to follow up on the modeling of Spitkovsky et al. (2002), which suggested a link between the rise times of Type I X-ray bursts and the spin rate of the star. The rise time of the lightcurve from a Type I X-ray burst depends on the latitude at which ignition occurs, the speed $v_{f}$ at which the flame front propagates across the star, and the star's inclination with respect to the observer. Spitkovsky et al. (2002) modeled flame spread across the surface of a rapidly rotating neutron star and showed that flame velocity $v_{f} \approx(g h)^{1 / 2} /\left(f t_{n}\right)^{k}$ where $g$ is the local gravitational acceleration, $h$ the scale height of the burning ocean, $t_{n}$ the timescale for nuclear burning and $f=4 \pi v_{s} \cos \theta$ the Coriolis parameter ( $\theta$ is the latitude). The power $k$ depends on the degree of frictional coupling between the top and bottom of the burning ocean, and is constrained to lie between 0.5 and 1 . Burst rise time therefore depends, at least in part, on spin rate. The other parameters can in many cases be constrained, by theory or observation (burst shape, for example, can pinpoint ignition latitude, Maurer et al., 2008). Isolating the effects of spin from the variation due to the other factors will still not be easy, but may be possible given the extensive archives of burst data that now exist. If the Spitkovsky et al. (2002) picture survives more rigorous modeling and test, then the fastest burst rise time should place at least an upper limit on the spin rate of the star.

Ideas like this, which are clearly more speculative, are certainly worth exploring given the potential payoff for the gravitational wave searches. If we could measure spin to within $\pm 5 \mathrm{~Hz}$ then four sources would move above the detectability threshold of Advanced LIGO provided that computational restrictions can be overcome. This is not an unreasonable hope: several ways of improving the computational situation were outlined in Watts et al. (2008). For the other sources better knowledge of spin alone is not sufficient and our knowledge of the orbital parameters must also improve. Fortunately there has been major progress in this area in recent years using optical/IR observations (for an overview see Cornelisse et al., 2008).

\section{Moving beyond spin balance}

The legitimacy of the spin balance scenario, particularly on short timescales, has always been a matter of debate. Accretion luminosity is observed to vary, and accretion 
episodes in many sources are transient. The timescales on which the various quadrupoles respond are also very poorly understood - within the r-mode scenario, for example, spin-down may be episodic.

Scenarios where the source is out of balance, and where gravitational wave torques might (at least temporarily) exceed accretion torques are attractive, as predicted signal could then be larger. The downside is that one must then take additional search parameters into account in the searches. We will start, however, by considering the best case scenario, assuming that we can track both spin and orbital parameters so that we only have to search a single template. One can write the change in spin rate $\dot{v}_{s}$ in terms of the moment of inertia $I$ and the other parameters defined previously.

$$
\begin{aligned}
I_{45} \dot{v}_{s}= & 1.4 \times 10^{-14} \mathrm{~Hz} / \mathrm{s} d_{\mathrm{kpc}}^{2} \\
& \times\left[F_{-8}\left(\frac{R_{10}^{3}}{M_{1.4}}\right)^{\frac{1}{2}}-11\left(\frac{v_{s}}{1 \mathrm{kHz}}\right)\left(\frac{h_{0}}{10^{-26}}\right)^{2}\right]
\end{aligned}
$$

where $I_{45}=I / 10^{45} \mathrm{~g} \mathrm{~cm}^{2}$ and $d_{\mathrm{kpc}}=d / 1 \mathrm{kpc}$. Below a certain flux the accretion term can be neglected and gravitational wave induced spin-down is the dominant term. Note that this equation neglects any other negative torques that may be present, such as those generated by magnetosphere/disk interaction, jets, or magnetic dipole spin-down. This means that we are considering the most optimistic scenario for gravitational wave emission.

To investigate the spin-down that would be associated with a detectable signal, we set $h_{0}$ equal to one of the detectability curves plotted in Fig. 1 (assuming a particular detector configuration and an integration time). Fig. 3 shows the spin-down rate that would be associated with

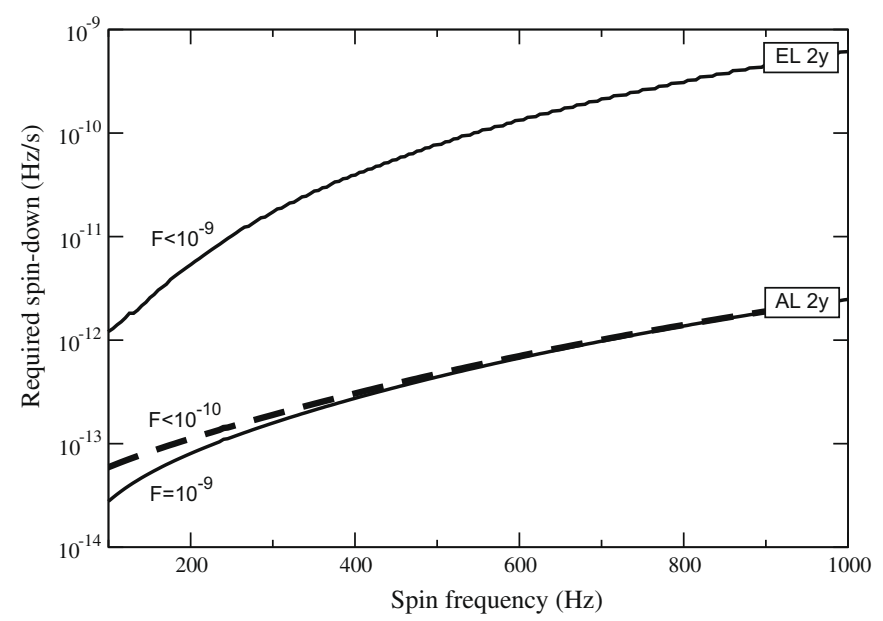

Fig. 3. For a signal to be detectable in the spin varying scenario, gravitational wave torque must exceed the accretion torque. This means that for a signal to be detectable there must be an associated spin-down (Eq. (4)). This plot shows the required spin-down for sources with low fluxes $F$ (in units of ergs $/ \mathrm{cm}^{2} / \mathrm{s}$ ) to be detectable in a 2-year integration by either Enhanced LIGO (EL) or Advanced LIGO (AL), assuming a typical source distance of $5 \mathrm{kpc}$. As in Fig. 1, this is the best case scenario, as it assumes perfect knowledge of spin and orbital parameters. such a signal, for a typical source distance of $d_{\mathrm{kpc}}=5$. For a 2-year integration, one would require $\left|\dot{v}_{s}\right| \sim 10^{-15} \mathrm{~Hz} / \mathrm{s}$ for there to be no additional template requirement. For both Enhanced and Advanced LIGO, however, a detectable signal from a low flux source would have a much larger spin-down. This means that it will be necessary to search spin derivative templates if spin cannot be tracked precisely (for burst oscillation sources, for example, or sources that are in quiescence). For the sources where we cannot track spin precisely we would also need to consider the likelihood of the source being in a spin-down episode when observed. Given this additional effect on detectability we will concentrate for now on the accreting millisecond pulsars.

We can compare the 'detectable' values of spin-down to the spin derivatives that have been reported in the literature for the accretion-powered millisecond pulsars (summarized in Watts et al., 2008). Pulse time of arrival analysis has led to claims of both spin-up and spin-down, with upper limits on $\left|\dot{v}_{s}\right| \sim 10^{-12} \mathrm{~Hz} / \mathrm{s}$. Timing analysis of these systems is a complex business due to the extreme pulse profile variability, and there is ongoing debate as to whether these spin derivatives are genuine or an artifact (Hartman et al., 2008). However, a concerted effort is underway to resolve these issues, and there is some hope of getting reliable values of spin derivatives in the near future.

If a spin-down larger than the detectability limit can be confirmed for any of the accreting millisecond pulsars, then simultaneous X-ray timing and gravitational wave searches would confirm whether gravitational waves provide the limiting torque. Given current schedules, there will be a gap in simultaneous X-ray timing as RXTE is scheduled to go offline in September 2009, in the middle of the Enhanced LIGO science run, and ASTROSAT, ${ }^{6}$ an Indian X-ray timing mission, may not launch until 2010. However, once Advanced LIGO starts to operate, ASTROSAT should be available to provide timing support. Certainly by the time that third generation gravitational wave detectors such as the Einstein Telescope ${ }^{7}$ (currently at the study stage) enter service, in conjunction with future X-ray timing missions, ${ }^{8}$ there is a very strong possibility of constraining pulsar torque models in this way.

Having some way of timing spin in quiescence would also be useful: there has been a suggestion that at least one of the accreting millisecond pulsars, SAX J1808.43658 , turns on as a radio pulsar in quiescence (Burderi et al., 2003; Campana et al., 2004). Searches for radio pulsations from this source at $1.4 \mathrm{GHz}$ have so far been unsuccessful (Gaensler et al., 1999; Burgay et al., 2003), as have searches at 2.0 and $4.6 \mathrm{GHz}$ with the Green Bank Telescope (Adrienne Juett, private communication). Nonethe-

\footnotetext{
${ }^{6} \mathrm{http}: / /$ meghnad.iucaa.ernet.in/ astrosat

${ }^{7}$ http://www.ego-gw.it/ILIAS-GW/FP7-DS/fp7-DS.htm

${ }^{8}$ Such as the proposed High Time Resolution Spectrometer (HTRS) on the International X-ray Observatory (IXO), or the Advanced X-ray Timing Array (AXTAR, Chakrabarty et al., 2008).
} 
less, future radio telescopes such as LOFAR, the Murchison Wide Field Array, and eventually the Square Kilometer Array may improve detection prospects.

\section{Conclusions}

With two exceptions (Abbott et al.,2007a,b), most of the effort in periodic gravitational wave searches to date has gone into searches for signals from isolated non-axisymmetric neutron stars. These include both targeted searches for known pulsars, and blind searches for unknown systems. While these blind surveys are computationally limited, searches for accreting neutron stars can be even more challenging in the absence of well-defined orbital parameters. Given the computational resources required, it is important that the searches are well motivated from an astrophysical standpoint. For isolated neutron stars, the spindown rate provides an indirect limit by assuming that all of the spindown is due to gravitational wave emission. However, the true amplitude is almost certainly much smaller than this; most of the spindown is well explained by electromagnetic braking (see, e.g. Palomba (2000) for estimates). For accreting neutron stars the indirect limit is provided by the spin balance argument and, if nature does employ this mechanism, the true amplitude is likely to be very close to this limit. Thus, the first direct detection of periodic gravitational radiation might very well be from accreting neutron stars. This is, however, going to be challenging, given the current state of our knowledge regarding spin and orbital parameters. It may be possible to reduce this uncertainty, but otherwise some out of the box thinking will be required.

One area that may lead to progress is a more in-depth consideration of prospects for indirect detection. Ideally what one would like is a signature of one of the gravitational wave emission mechanisms that could be detected more easily in electromagnetic wavebands. One possibility is to look for the signature of the mechanism that generates the quadrupole. Ushomirsky et al. (2000), for example, point out that a quadrupole induced by temperature asymmetries in the deep crust may lead to detectable asymmetries in surface X-ray emission, provided that the gradients do not wash out as radiation escapes through the ocean and atmosphere. Payne and Melatos (2006b) have also considered the implications of a magnetic mountain on the observable properties of Type I X-ray bursts and burst oscillations. Ideas along these lines merit more detailed study.

A second possibility for indirect detection is to look for the effect of angular momentum loss associated with gravitational wave emission by monitoring spin evolution. As pointed out by Chakrabarty (2008), X-ray timing (for accreting millisecond pulsars) is able to detect very small changes in spin rate, and is potentially much more sensitive to gravitational wave spin-down than the gravitational wave detectors themselves. Measuring spin variations in outburst is complicated by uncertainties associated with pulse profile variability, but spin changes between out- bursts may provide stronger constraints. The main issue is then whether it is possible to make an unambiguous identification of the cause of the spin-down, given the other negative torque mechanisms that may operate.

We may also want to consider a change of emphasis in our theoretical modeling. Rather than focusing on what might be detectable, theorists should perhaps consider what upper limits would be most physically interesting. In the spin balance scenario there are several sources that could be detectable by Advanced LIGO if we searched smaller frequency bands: so which bands should we search? The strongest limits on gravitational wave amplitude will be obtained if we restrict searches to a low frequency band around the sweet spots of the detectors. The limits on quadrupole moment $Q$, however, will be tighter if we focus on a higher frequency band. Which of these options would make the biggest difference to our understanding of neutron star physics or binary evolution? Is it better to search at the dominant r-mode frequency (four thirds the spin frequency) or the fixed quadrupole frequency (twice the spin frequency)? Should we be focusing our attention on spindown scenarios rather than spin-balance scenarios? Certainly for the accreting millisecond pulsars, where simultaneous X-ray timing may enable spin tracking, this latter scenario is much more attractive and (given current estimates of spin variation) could lead to physically meaningful constraints on torque models. We cannot hope to answer all of these questions in such a short paper. However, these are issues that the community should be debating if we are to make the most of the data that the gravitational wave observatories obtain in the near future.

\section{Acknowledgments}

A.L.W. thanks COSPAR and the Leids KerkhovenBosscha Fonds (LKBF) for financial support to attend the 37th COSPAR meeting in Montreal. We would also like to thank Lars Bildsten, Deepto Chakrabarty, Jason Hessels, Adrienne Juettt and Bernard Schutz for useful discussions that informed this work.

\section{References}

Abbott, B., Abbott, R., Adhikari, R., et al. Searches for periodic gravitational waves from unknown isolated sources and Scorpius X-1: results from the second LIGO science run. Phys. Rev. D 76, 82001, $2007 \mathrm{a}$.

Abbott, B., Abbott, R., Adhikari, R., et al. Upper limit map of a background of gravitational waves. Phys. Rev. D 76, 082003, 2007b.

Andersson, N., Kokkotas, K.D., Stergioulas, N. On the relevance of the rmode instability for accreting neutron stars and white dwarfs. Astrophys. J. 516, 307-314, 1999.

Andersson, N., Jones, D.I., Kokkotas, K.D., Stergioulas, N. R-Mode runaway and rapidly rotating neutron stars. Astrophys. J. 534, L75L78, 2000.

Andersson, N., Jones, D.I., Kokkotas, K.D. Strange stars as persistent sources of gravitational waves. Mon. Not. Roy. Astron. Soc. 337, 1232-1244, 2002.

Bildsten, L. Gravitational radiation and rotation of accreting neutron stars. Astrophys. J. 501, L89-L93, 1998. 
Bondarescu, R., Teukolsky, S.A., Wasserman, I. Spin evolution of accreting neutron stars: nonlinear development of the r-mode instability. Phys. Rev. D 76, 64019, 2007.

Burderi, L., di Salvo, T., d'Antona, F., Robba, N.R., Testa, V. The optical counterpart to SAX J1808.4-3658 in quiescence: evidence of an active radio pulsar? Astron. Astrophys. 404, L43-L46, 2003.

Burgay, M., Burderi, L., Possenti, A., D’Amico, N., Manchester, R.N., Lyne, A.G., Camilo, F., Campana, S. A search for pulsars in quiescent soft X-ray transients. I. Astrophys. J. 589, 902-910, 2003.

Campana, S., d'Avanzo, P., Casares, J., et al. Indirect evidence of an active radio pulsar in the quiescent state of the transient millisecond pulsar SAX J1808.4-3658.. Astrophys. J. 614, L49-L52, 2004.

Chakrabarty, D. The spin distribution of millisecond X-ray pulsars. ArXiv eprints, 0809.4031, 2008.

Chakrabarty, D., Ray, P.S., Strohmayer, T.E., for the AXTAR Collaboration. The Advanced X-ray Timing Array (AXTAR). ArXiv eprints, 0809.4029, 2008.

Cook, G.B., Shapiro, S.L., Teukolsky, S.A. Recycling pulsars to millisecond periods in General Relativity. Astrophys. J. 423, L117-L120, 1994.

Cornelisse, R., Casares, J., Muñoz-Darias, T., Steeghs, D., Charles, P., Hynes, R., O'Brien, K., Barnes, A. An overview of the Bowen Survey: detecting donor star signatures in low mass X-ray binaries. In: Bandyopadhyay, R.M., Wachter, S., Gelino, D., Gelino, C.R. (Eds.), A Population Explosion: The Nature \& Evolution of X-ray Binaries in Diverse Environments, American Institute of Physics Conference Series, vol. 1010, pp. 148-152, May 2008.

Cutler, C. Gravitational waves from neutron stars with large toroidal B fields. Phys. Rev. D 66, 084025, 2002.

Gaensler, B.M., Stappers, B.W., Getts, T.J. Transient radio emission from SAX J1808.4-3658. Astrophys. J. 522, L117-L119, 1999.

Hartman, J.M., Patruno, A., Chakrabarty, D., Kaplan, D.L., Markwardt, C.B., Morgan, E.H., Ray, P.S., van der Klis, M., Wijnands, R. The long-term evolution of the spin, pulse shape, and orbit of the accretionpowered millisecond pulsar SAX J1808.4-3658. Astrophys. J. 675, 1468-1486, 2008.

Haskell, B., Jones, D.I., Andersson, N. Mountains on neutron stars: accreted versus non-accreted crusts. Mon. Not. Roy. Astron. Soc. 373, 1423-1439, 2006.

Haskell, B., Samuelsson, L., Glampedakis, K., Andersson, N. Modelling magnetically deformed neutron stars. Mon. Not. Roy. Astron. Soc. 385, 531-542, 2008.

Hessels, J.W.T., Ransom, S.M., Stairs, I.H., Freire, P.C.C., Kaspi, V.M., Camilo, F. A radio pulsar spinning at $716 \mathrm{~Hz}$. Science 311, 1901-1904, 2006.
Heyl, J.S. Low-mass X-ray binaries may be important laser interferometer gravitational-wave observatory sources after all. Astrophys. J. 574, L57-L60, 2002.

Jaranowski, P., Królak, A., Schutz, B.F. Data analysis of gravitationalwave signals from spinning neutron stars: the signal and its detection. Phys. Rev. D 58, 63001, 1998.

Katz, J.I. Do AM Hercules white dwarfs have toroidal internal fields? MNRAS 239, 751-758, 1989.

Kulkarni, A.K., Romanova, M.M. Accretion to magnetized stars through the Rayleigh-Taylor instability: global 3D simulations. Mon. Not. Roy. Astron. Soc. 386, 673-687, 2008.

Lattimer, J.M., Prakash, M. Neutron star observations: prognosis for equation of state constraints. Phys. Rep. 442, 109-165, 2007.

Levin, Y. Runaway heating by R-modes of neutron stars in low-mass Xray binaries. Astrophys. J. 517, 328-333, 1999.

Maurer, I., Watts, A.L. Ignition latitude and the shape of Type I X-ray bursts. MNRAS 383, 387-398, 2008.

Melatos, A., Payne, D.J.B. Gravitational radiation from an accreting millisecond pulsar with a magnetically confined mountain. Astrophys. J. 623, 1044-1050, 2005.

Nayyar, M., Owen, B.J. R-Modes of accreting hyperon stars as persistent sources of gravitational waves. Phys. Rev. D 73, 084001, 2006.

Palomba, C. Pulsars ellipticity revised. Astron. Astrophys. 354, 163-168, 2000.

Payne, D.J.B., Melatos, A. Frequency spectrum of gravitational radiation from global hydromagnetic oscillations of a magnetically confined mountain on an accreting neutron star. Astrophys. J. 641, 471-478, 2006a.

Payne, D.J.B., Melatos, A. Magnetic burial and the harmonic content of millisecond oscillations in thermonuclear X-ray bursts. Astrophys. J. 652, 597-602, 2006b.

Spitkovsky, A., Levin, Y., Ushomirsky, G. Propagation of thermonuclear flames on rapidly rotating neutron stars: extreme weather during Type I X-ray bursts. Astrophys. J. 566, 1018-1038, 2002.

Ushomirsky, G., Cutler, C., Bildsten, L. Deformations of accreting neutron star crusts and gravitational wave emission. Mon. Not. Roy. Astron. Soc. 319, 902-932, 2000.

Vigelius, M., Melatos, A. Three-dimensional stability of magnetically confined mountains on accreting neutron stars. MNRAS 386, 1294 1308, 2008.

Wagoner, R.V. Conditions for steady gravitational radiation from accreting neutron stars. Astrophys. J. 578, L63-L66, 2002.

Watts, A.L., Krishnan, B., Bildsten, L., Schutz, B.F. Detecting gravitational wave emission from the known accreting neutron stars. Mon. Not. Roy. Astron. Soc. 389, 839-868, 2008. 